\title{
Characterization of a calcium/calmodulin- regulated SR/CAMTA gene family during tomato fruit development and ripening
}

\author{
Tianbao Yang*, Hui Peng, Bruce D Whitaker and William S Conway
}

\begin{abstract}
Background: Fruit ripening is a complicated development process affected by a variety of external and internal cues. It is well established that calcium treatment delays fruit ripening and senescence. However, the underlying molecular mechanisms remain unclear.

Results: Previous studies have shown that calcium/calmodulin-regulated SR/CAMTAs are important for modulation of disease resistance, cold sensitivity and wounding response in vegetative tissues. To study the possible roles of this gene family in fruit development and ripening, we cloned seven SR/CAMTAS, designated as SISRS, from tomato, a model fruit-bearing crop. All seven genes encode polypeptides with a conserved DNA-binding domain and a calmodulin-binding site. Calmodulin specifically binds to the putative targeting site in a calcium-dependent manner. All SISRs were highly yet differentially expressed during fruit development and ripening. Most notably, the expression of SISR2 was scarcely detected at the mature green and breaker stages, two critical stages of fruit development and ripening; and SISR3L and SISR4 were expressed exclusively in fruit tissues. During the developmental span from 10 to 50 days post anthesis, the expression profiles of all seven SISRs were dramatically altered in ripening mutant rin compared with wildtype fruit. By contrast, only minor alterations were noted for ripening mutant nor and $\mathrm{Nr}$ fruit. In addition, ethylene treatment of mature green wildtype fruit transiently stimulated expression of all SISRs within one to two hours.

Conclusions: This study indicates that SISR expression is influenced by both the Rin-mediated developmental network and ethylene signaling. The results suggest that calcium signaling is involved in the regulation of fruit development and ripening through calcium/calmodulin/SISR interactions.
\end{abstract}

\section{Background}

Fleshy fruits, a significant part of the human diet, provide fiber, minerals and various nutraceuticals, and promote human health. Fruit quality and postharvest shelf life are dependent upon the control of ripening. Fruit ripening is a complex, genetically programmed process. Classically, fruits are grouped into two ripening types, climacteric and non-climacteric. The ripening of climacteric fruits such as tomato and apple, usually is triggered by biosynthesis of the gaseous hormone ethylene. In contrast, the ripening of non-climacteric fruits, such as strawberry and grape is mediated by an ethylene-independent process with little changes in respiration rate

\footnotetext{
* Correspondence: tianbao.yang@ars.usda.gov
Food Quality Laboratory, Plant Science Institute, USDA-ARS, Beltsville 20705,

* Correspondence: tianbao.yang@ars.usda.gov
Food Quality Laboratory, Plant Science Institute, USDA-ARS, Beltsville 20705, $M D$, USA
} 
second messenger (reviewed in [13-19]). Cellular calcium changes can be sensed and interpreted by calciumbinding proteins that function as signal sensors [20]. Calmodulin is one of the most well characterized calcium-sensors and functions as a modulator to other target proteins (reviewed in [21-23]). These proteins play roles in metabolism, ion transport, transcriptional regulation, protein phosphorylation, and other critical functions. Recently, a calcium/calmodulin-regulated SR/ CAMTA transcription factor family has been shown to play an important role in the plant response to abiotic and biotic stresses. First identified in plants [24], SR/ CAMTAs are present in all plant and animal species surveyed to date. In plants, SR/CAMTAs show differential responses to developmental signals and a variety of environmental signals. For instance, the tobacco ortho$\log N t E R 1$ is an ethylene-responsive gene and highly expressed in senescing flowers and leaves [24]. Expression of the tomato ortholog ER66 is stimulated by ethylene, and is higher in fruit tissues at the red ripe stage than those at the mature green stage [25]. In Arabidopsis, six AtSRs differentially respond to a variety of external signals, such as cold, wounding and drought, as well as hormonal signals like ethylene and ABA [26-28]. The knockout of AtSR1 led to increased accumulation of salicylic acid and enhanced disease resistance [29]. Salicylic acid can reduce the expression of ACC synthase and affect ethylene biosynthesis [30,31]. AtSR1/CAMTA3 and AtSR2/CAMTA1 are also important for plant tolerance to low temperature [32]. Knockout of those genes significantly reduced cold tolerance. The genes affected by AtSR1/CAMTA3 include PR genes, expansin, beta1,3-glucanase, phospholipase A2, accelerated cell death protein 6 , and senescence associated gene 21 [33]. The SR/CAMTAs' primary target of CGCG cis-element has been suggested to be the major calcium-regulated ciselement [34] and the rapid wounding responsive element [35]. Therefore SR/CAMTAs may sit at the crossroads where calcium signaling intersects the ethylene, salicylic acid, wounding and cold signal transduction pathways. All of these signaling pathways have a major impact on fruit ripening and quality.

To investigate the role of calcium-regulated SR/ CAMTA in fruit ripening, we selected tomato, a model fruit-bearing crop, because of its well characterized molecular basis of fruit ripening and its economic importance. Tomato (Solanum lycopersicum) is one of the most important horticultural crops. It has become an excellent model to study fleshy fruit development and ripening because highly developed genetic and molecular toolkits are available $[3,36]$. Single gene mutants representing ripening associated phenotypes have been well characterized (reviewed in [37]). These include the recessive ripening mutant rin (ripening- inhibitor), and dominant ripening mutants Nor (nonripening), $\mathrm{Nr}$ (never-ripe), $\mathrm{Cnr}$ (colorless non-ripening) and $\mathrm{Gr}$ (green-ripe) [38-41]. Of them, the Rin, and Nor genes encode a MADS-box and a NAC-domain transcription factor, respectively, and act upstream of crucial ripening activities including ethylene production, suggesting that they regulate ethylene-independent ripening processes [39]. In contrast, $\mathrm{Nr}$ is directly involved in ethylene-dependent ripening because $\mathrm{Nr}$ encodes an ethylene receptor [38]. Here we report the isolation of seven SlSRs, the SR/CAMTA orthologs in tomato and characterization of their expression patterns during fruit development in wildtype fruits and ripening mutants, as well their response to ethylene treatment.

\section{Results}

\section{Seven SR/CAMTAs are expressed in tomato}

There are six SR/CAMTAs (AtSR1-6) in the Arabidopsis genome and all of them are expressed in tissues [26]. To clone the tomato counterparts, the amino acid sequences of the six Arabidopsis SR/CAMTAs were used to search GenBank http://www.ncbi.nlm.nih.gov and tomato databases on the SOL Genomics Network http://solgenomics.net. There were about 50 EST clones and 16 Unigenes showing linear homology to AtSR1-6, but none of them included a full-length coding region. Analysis of the ESTs and Unigenes indicated that there are seven putative SR/CAMTAs, designated as SlSRs, expressed in tomato. To obtain the full length cDNAs, a mixture of total RNAs from various tissues and fruit pericarp at several developmental stages was used for reverse transcription and amplification with gene specific primers (Table 1). The primer sets for cloning three of them, SISR1, SlSR2 and SlSR3, were designed directly from the available Unigenes/ESTs based on the amino acid sequence alignment with Arabidopsis AtSRs in the $\mathrm{N}$-terminal and $\mathrm{C}$-terminal regions. For the remaining four genes, their missing 5' and 3' ends were obtained using 5 ' and/or 3' RACE. The gene specific primer pairs were further utilized to amplify their full length coding regions. Three of the four SISRs were named as SISR1L, SISR2L and SISR3L because they showed high homology to SlSR1, SlSR2 and SlSR3, respectively. However, SISR4 had no close relationship to any of the other six. All SlSR encoded polypeptides include the SR/CAMTA structural features: a conserved DNA-binding domain in the N-terminal portion, a calmodulin-binding domain in the C-terminal portion, and ankyrine repeats in the middle, suggesting that tomato SlSRs are true orthologs of the $S R / C A M T A$ transcription factor family. As shown in Additional File 1, the deduced amino acid sequences of the seven SlSRs have $45-76 \%$ and $30-65 \%$ overall similarity and identity, respectively. However, there are over $80-93 \%$ similarity and $63-88 \%$ identity of amino acid 
Table 1 Primers Used for Gene Cloning and qPCR

\begin{tabular}{|c|c|}
\hline SISR1-P1* & ATGGCAGACAGTAGGCGTTA \\
\hline SISR1-P2* & TCAAGGTGCTGTAGGCATAAAA \\
\hline SISR1L-P1* & ATGGACATAACACAGATATTATCCG \\
\hline SISR1L-P2* & TTATTCAAATGCTATAGACATGAAAGTA \\
\hline SISR2-P1* & ATGGCAGAATCAGGATACAACACA \\
\hline SISR2-P2* & TTAGACATGTCCATGAGCAGTTG \\
\hline SISR2L-P1* & ATGGCGGAATCAGGATATGATATT \\
\hline SISR2L-P2* & CTAGATGGATGATTGACTGACCT \\
\hline SISR3-P1* & ATGGAAAGCAACAGAGCAGGAC \\
\hline SISR3-P2* & CTAGTTATCAGGATTGATAAGCCTT \\
\hline SISR3L-P1* & ATGGAAAGTAGCGTATCAGGACGA \\
\hline SISR3L-P2* & TTAGTCCATCTCAGTGTCAGGATTG \\
\hline SISR4-P1* & ATGGCAGTAGATCTTGAACAGATA \\
\hline SISR4-P2* & CTAAACTGGTGGTGATGACCTA \\
\hline SISR1-A** & CAGAAGGCTCTGGAAAGGGTAA \\
\hline SISR1-B** & AAATCAACTGCTTCCGCTGAGT \\
\hline SISR1L-A** & AAGTGAAGCAGGATGGTCCAAT \\
\hline SISR1L-B** & ATTGGCTAATCAAGGGGGAAAA \\
\hline SISR2-A** & CTITGCACCCAGTTTTAAATGC \\
\hline SISR2-B** & GTAACAGCTCCAGCAAATGCAC \\
\hline SISR2L-A** & AAATGCTGAAGCCCTGAATTGT \\
\hline SISR2L-B** & ATTGGCTAATCAAGGGGGAAAA \\
\hline SISR3-A** & ACACAATAATGCTTCGCTGGAA \\
\hline SISR3-B** & GGGGCAATATCACAATGCTTTT \\
\hline$\overline{S I S R 3 L-A^{* *}}$ & GCAATGGAAAGTAGCGTATCAGG \\
\hline SISR3L-B** & GGCAAGTITACAGGCTTGACATT \\
\hline SISR4-A** & TTCTGGCCAAAATCCTCGTAAT \\
\hline SISR4-B** & TTGTGAAAATGCAGGCTCTTGT \\
\hline $\mathrm{E}_{4} \mathrm{~A}^{* *}$ & ACCAGCAATATCTAGAGAAGGGTG \\
\hline$E 4-B^{* *}$ & ATCATTGTCATGTTTATTCAAATTTAAAG \\
\hline Actin- $A^{* *}$ & GAAATAGCATAAGATGGCAGACG \\
\hline Actin- $B^{* *}$ & ATACCCACCATCACACCAGTAT \\
\hline
\end{tabular}

*Primers used for cloning; **Primers used for qPCR

sequences in the $\mathrm{N}$-terminal DNA-binding domain and the C-terminal calmodulin-binding domain. The phylogenetic relationship of SISR encoded polypeptides and Arabidopsis AtSRs was further analyzed (Figure 1). SISR1 is closely related to AtSR1, SISR2 and SISR2L are in the same clade with AtSR5, and SISR3 and SISR3L are very similar to AtSR3 and AtSR6. In contrast, SISR1L has a weak homology to AtSR2, AtSR4 and SISR1, whereas SISR4 is not closely related to any of them, suggesting that SlSR4 is a relatively new member of the tomato $S R / C A M T A$ gene family.

\section{All SISRs encode calcium/calmodulin-binding proteins}

All SR/CAMTA proteins reported thus far are known to be calcium/calmodulin-binding proteins. To ascertain

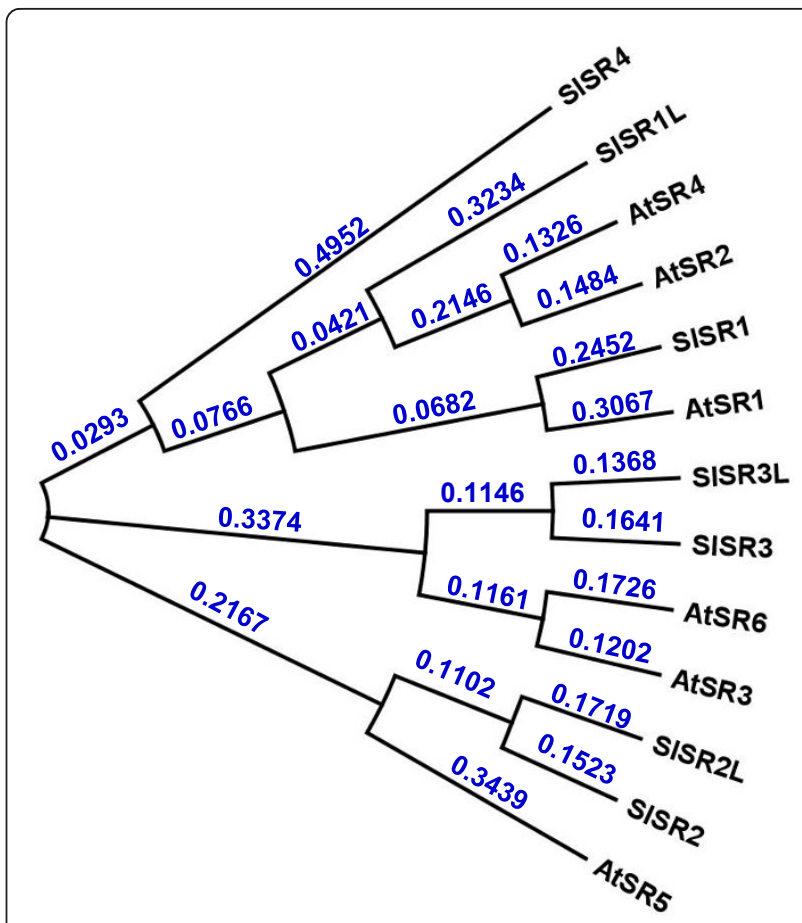

Figure 1 Phylogenetic analysis of amino acid sequences encoded by seven tomato SISRs and SR/CAMTA orthologs from Arabidopsis thaliana. The corresponding GenBank cDNA accession numbers or gene identification numbers are as follows: SISR1, GU170838; SISR1L, JN558810; SISR2, JN566047; SISR2L, JN566048; SISR3, JN566049; SISR3L, JN566051; SISR4, JN566050; AtSR1, AT2G22300; AtSR2, At5G09410; AtSR3, At3G16940; AtSR4, At5G64220; AtSR5, At1G67310; AtSR6, At4G16150. Numbers at tree nodes indicate the genetic distances in substitutions per site.

whether tomato SlSRs encode calcium/calmodulin-binding proteins, their putative calmodulin-binding regions were aligned with the corresponding regions in Arabidopsis AtSRs (Figure 2A). The corresponding sites in AtSRs are well characterized calcium/calmodulin-bining regions and share very high homology with their counterparts in the SISRs (overall > 90\% amino acid sequence identity). Within this region, SISR1, SISR1L, SISR2, SISR2L and SISR4 have almost the same amino acid sequence as AtSR1, and SISR3 and SISR3L show high similarity to AtSR3. All can form a basic amphipathic $\alpha$-helix structure [42], which can be recognized by calmodulin. We selected SISR3 as an example to show a basic amphipathic $\alpha$-helix structure projection, where the hydrophobic half is on the left side, and the basic hydrophilic half is on the right side (Figure 2B). Furthermore, IPTG-induced bacterial expression of the full-length coding region of SlSR3 fused with a $\mathrm{His}_{6}$-tag yielded a recombinant SISR3 band with a molecular mass of $\sim 105 \mathrm{kDa}$, as determined by SDS-PAGE (Figure 2C). A calmodulin overlay assay showed that calmodulin binds SISR3 in the presence of calcium, but not in the 


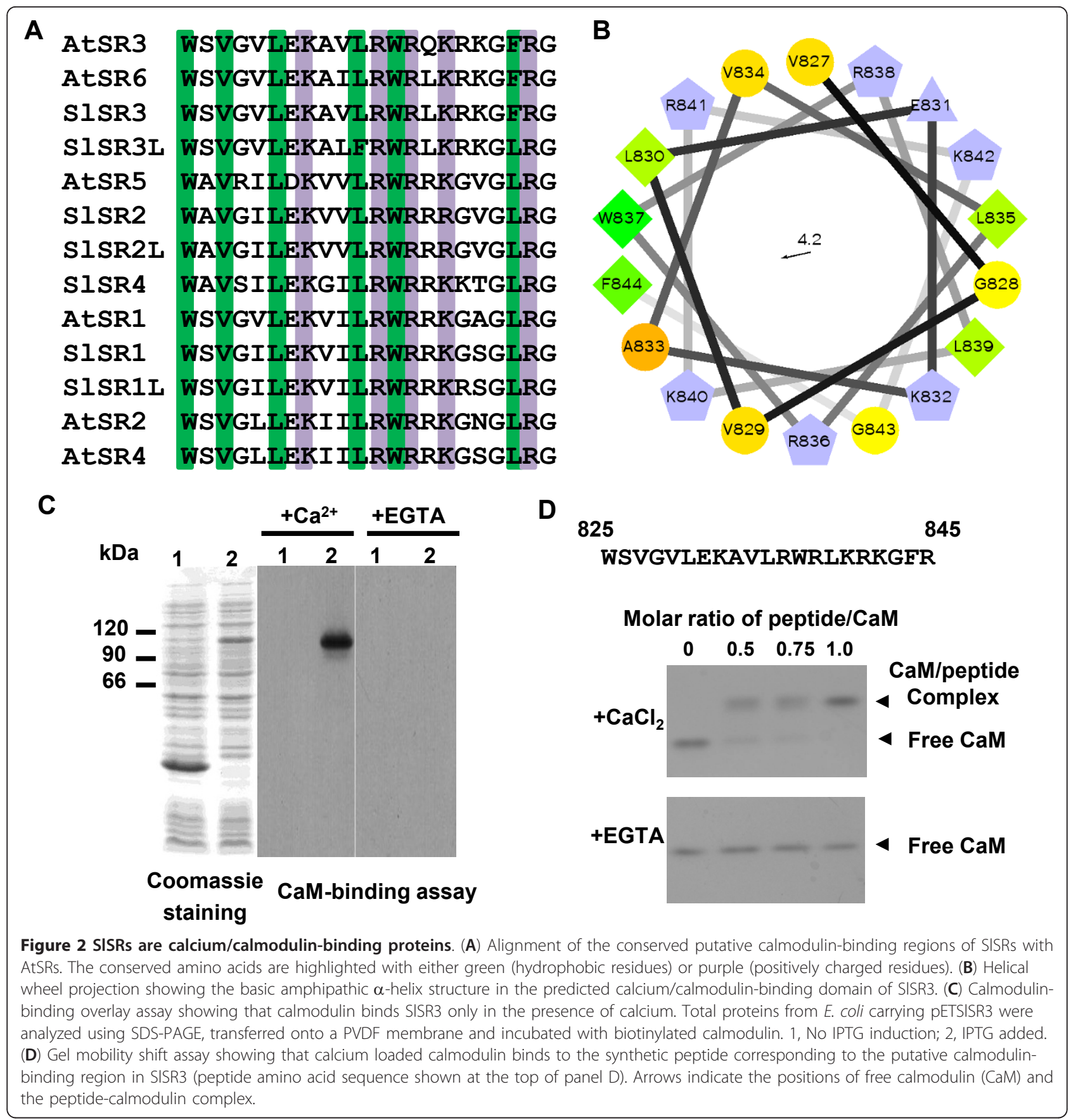

presence of EGTA, a calcium chelator. Finally, a gelmobility shift assay was used to confirm calmodulin binding specifically to the putative calmodulin-binding site in SISR3 (Figure 2D). A synthesized peptide corresponding to the 21-amino acid calmodulin-binding region of SISR3 was incubated with calmodulin in the presence of calcium or EGTA. After separation by native PAGE, the calmodulin-peptide complex was observed in the presence of calcium. The intensity of the complex band increased following an increase in the peptide-calmodulin ratio. By contrast, only a free calmodulin band appeared in the presence of EGTA. To investigate whether all seven SISRs are calcium/calmodulin-binding proteins, three additional peptides corresponding to the putative calmodulin-binding regions in SISR1, SISR2/SISR2L, and SISR4 were synthesized and assayed. The gel mobility shift assays showed that calmodulin binds to each of the three peptides only in the presence of calcium (Additional File 2). Since the corresponding region of SISR1 and SISR3L to SISR1L and 
SISR3, respectively contains just one or two conservative amino acid substitution(s), the results indicate that all SISRs are calcium/calmodulin-binding proteins.

\section{SISRs are differentially expressed in tomato tissues and during fruit development}

We cloned seven SlSRs from the pooled cDNA derived from an array of tomato tissues, indicating that there are seven expressed tomato $S R / C A M T A$ s. To investigate the temporal and spatial expression profiles of these SISRs, the relative levels of their transcripts in various tissues and in fruit at different developmental stages were quantified using RT-qPCR. All expression levels as related to the expression of actin gene are shown in Figure 3. Overall, the expression levels for all SlSRs were low in leaves and flowers, and relatively high in fruit. Some genes, such as SISR2, SISR2L and SlSR3, were highly expressed in roots. All the SlSRs exhibited differential expression patterns in various tissues and at various fruit development stages.

During fruit development and ripening, SISR1 showed three peaks of expression, which appeared at 10 DPA, the mature green stage and the red ripe stage. SISR1L transcript increased gradually after 25 DPA during fruit enlargement and ripening, while SISR $2 L$ had a bell curve expression pattern, with the peak around the mature green stage. SISR3 expression levels exhibited the least variation among different stages (less than two fold difference) with the highest expression at the red ripe stage. Expression of SISR3L and SISR4 was shown to be fruit specific. Moreover, SlSR4 was expressed only in turning and orange fruit, while SISR3L transcript levels peaked at the breaker stage. The most interesting expression pattern was that of SlSR2. It was highly expressed in fruit at all developmental stages except mature green and breaker, the earliest stages of ripening, when expression was undetectable. To exclude the possibility that the unique SISR2 expression pattern is unique to the Rutgers cultivar, we examined fruit of the cultivars Moneymaker and Alisa Craig as well, and found that SlSR2 transcript was scarcely detectable at the mature green stage, too (data not shown). Overall, these results indicate that the seven SlSRs are differentially expressed during fruit development and ripening.

\section{Expression of SISRs in fruit of tomato ripening mutants}

In the earliest stages of ripening, tomato fruit transition from mature green, when maximum size is attained but the skin and flesh remain green, to breaker, when color change and other aspects of ripening are first evident. Several tomato spontaneous ripening mutants, including rin, Nor and $N r$, have normal fruit size but fail to ripen. To determine whether SISRs have any relationship to the defined fruit ripening genes that are deficient in these mutants, we compared expression levels of the seven SlSRs in wildtype fruit at the mature green and breaker stages with those in mutant fruit at the chronologically equivalent stages 45 and 50 DPA, respectively (Figure 4).

At the mature green stage/45 DPA, expression levels of SISR1, SISR1L and SISR2L were consistently higher in the mutant compared with wildtype fruit. Specifically, SISR $1 L$ and SISR2L expression was about twofold higher in rin, and about 50\% higher in Nor and $N r$, whereas for SISR 1 transcript was roughly $50 \%$ more abundant in all three mutants. For both SISR3L and SISR4, increased expression relative to wildtype was noted only in the rin mutant. In contrast, expression of SISR3 was lower in rin and Nor, while in $\mathrm{Nr}$ it was twofold higher than in wildtype fruit. The most dramatic changes were found for SlSR2 and SlSR4. SlSR2 was not expressed in wildtype fruit, but was highly expressed in rin, as well as in $\mathrm{Nr}$ and $\mathrm{Nor}$ (about $40 \%$ relative to rin). SlSR4 expression was fivefold higher in rin compared with wildtype fruit.

At the breaker stage/50 DPA, the most obvious trend was that, except for SISR4, the expression levels of all SlSRs was significantly altered in rin compared with wildtype fruit (Figure 4). SlSR3L was completely suppressed in rin fruit, whereas expression levels of the other five genes were at least fivefold higher than in wildtype. In contrast, with the exception of SISR2, differences in SlSR expression levels relative to those in wildtype fruit were much less pronounced in Nor and $\mathrm{Nr}$ fruit. Similar to the expression profiles at the mature green stage, SISR2 transcript was not detectable in wildtype fruit, but was abundant in fruit of all three mutants, with the highest level in rin. In contrast, SlSR4 expression was not significantly different in fruit of wildtype and the three ripening mutants. Thus, overall, the rin mutation had by far the most marked influence on SISR gene expression at the mature green and breaker equivalent stages of fruit development.

We further investigated whether expression of SISR genes differs in rin and wildtype fruit prior to the mature green stage, comparing SISR mRNA levels at 10, 20 and 30 DPA (Figure 5). All the SlSRs except SlSR4 had lower expression in rin fruit before 45 DPA, which is equivalent to the mature green stage in wildtype fruit. By contrast, SlSR4 showed significantly higher expression in rin than in wildtype fruit at 10,20,30, and 45 DPA, and then declined below the wildtype level at 50 DPA (equivalent to breaker stage), in part due to a fivefold increase in SlSR4 transcript in wildtype. Thus, SISR expression patterns are altered in rin mutant fruit during development, with dramatic changes relative to the patterns in wildtype fruit occurring at the mature green and breaker equivalent stages. These differences in SISR expression are most likely related to fruit ripening rather 

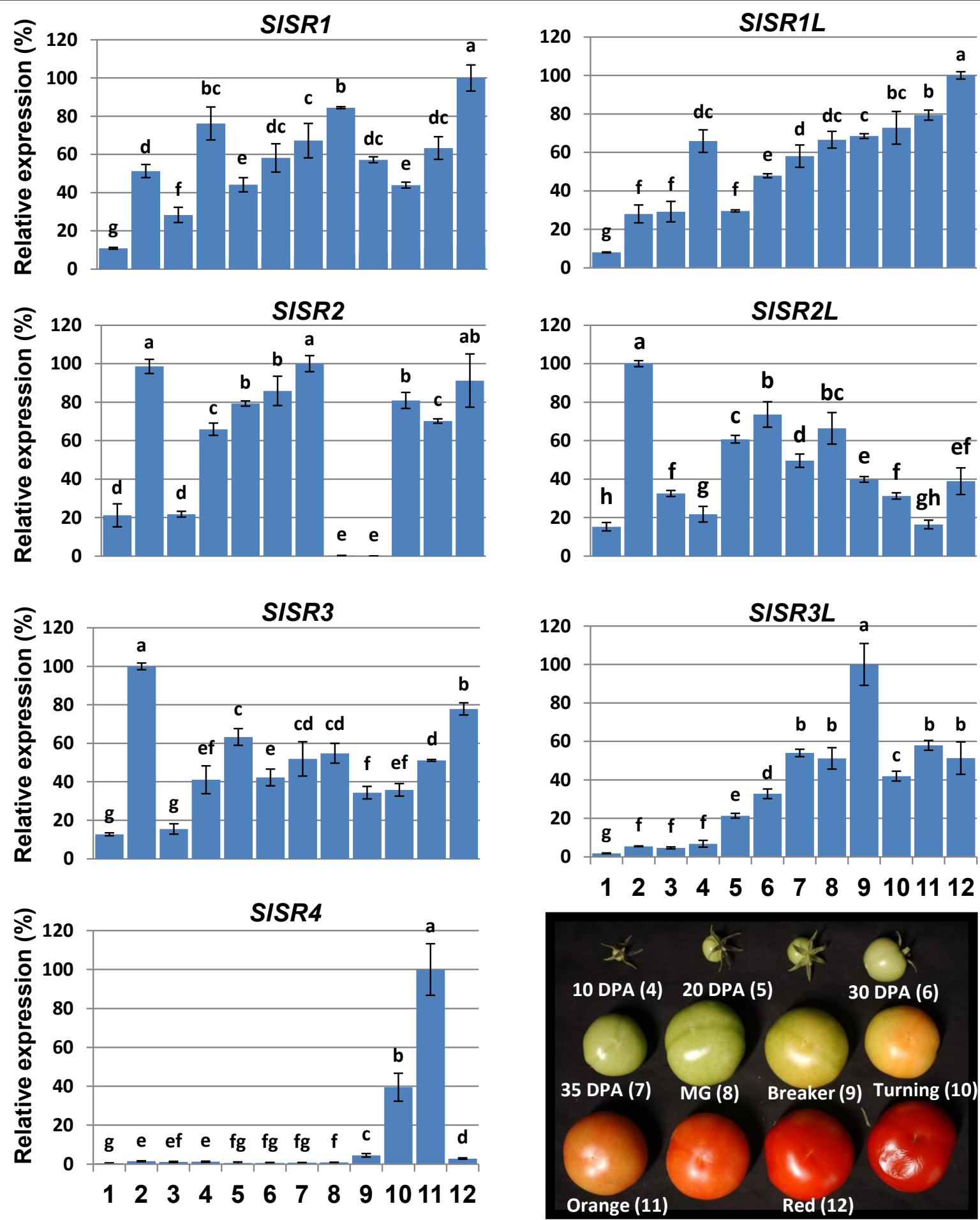

Figure 3 Expression patterns of SISR genes in different tissues and during fruit development. Transcription levels of SISRS were investigated by RT-qPCR. Relative gene expression levels (highest value $=100 \%$ ) are shown after normalization with actin transcript values. Error bars represent standard error of the mean. For each gene, different letters indicate significant differences among mean values $(P<0.05 ; t$-test). Numbers on the x-axis represent the following: two-week old seedling leaves (1) and roots (2), flowers (3), fruit at 10, 20, 30, and 35 DPA (4-7), and fruit at the mature green, breaker, turning, pink and red stages (8-12). The RT-qPCR analyses were repeated at least three times in three independent experiments. Photos of fruit at the nine developmental stages assessed (4-12; 10 DPA to red ripe) are shown on the bottom right. 


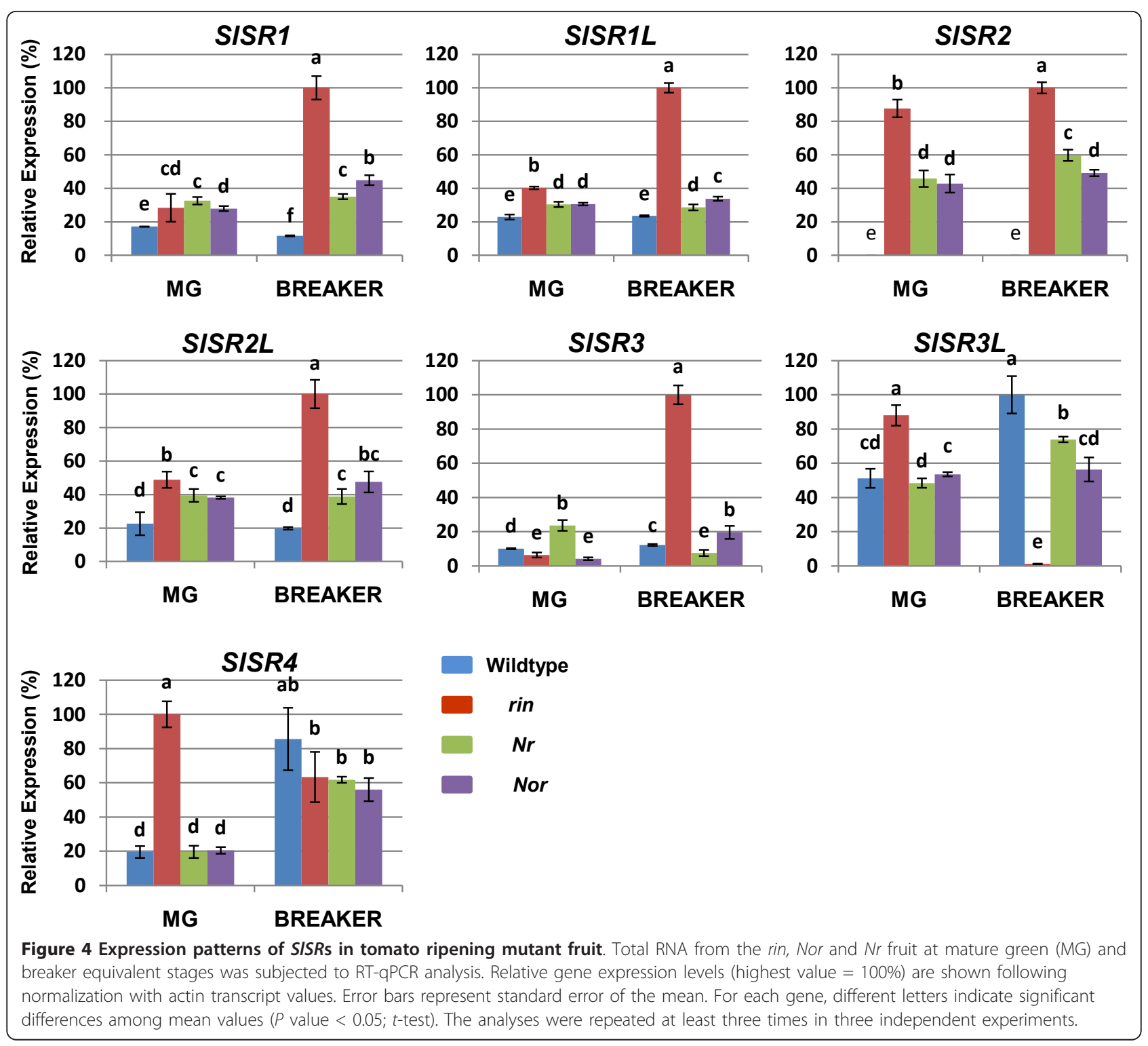

than expansion growth since rin yields normal sized fruit.

SISRs are early ethylene responsive genes

Ethylene is the major phytohormone controlling tomato fruit ripening and senescence. In tobacco and Arabidopsis, SR/CAMTAs are responsive to ethylene treatment $[24,26,28]$. To determine if all SISR genes in tomato fruit are responsive to ethylene, we selected mature green stage fruit for ethylene treatment because little endogenous ethylene is produced at this stage. As shown in Figure 6, expression of all SlSRs was transiently up-regulated by exogenous ethylene treatment. The ethylene stimulation or induction for all the genes was very rapid, usually within one hour after treatment. In particular, SISR2, which is not expressed at the mature green stage, was induced by 100 ppm ethylene within one hour after treatment. SISR1 was stimulated about fourfold by ethylene and peaked two hours after ethylene treatment. The remaining genes were stimulated about twofold by ethylene within one hour and peaked at one or two hours after treatment. For each of the SlSRs with the exception of SlSR2, the stimulation of expression by ethylene was transient, i.e. after four hours transcript levels were the same or lower than those at zero hours. By contrast, SlSR2 transcript declined about threefold from one to two hours but then increased two fold from two to four hours. E4, a well characterized ethylene responsive gene [43], was induced dramatically by ethylene, indicating that the ethylene treatment was effective. These results suggest that SlSRs are early ethylene responsive genes. 


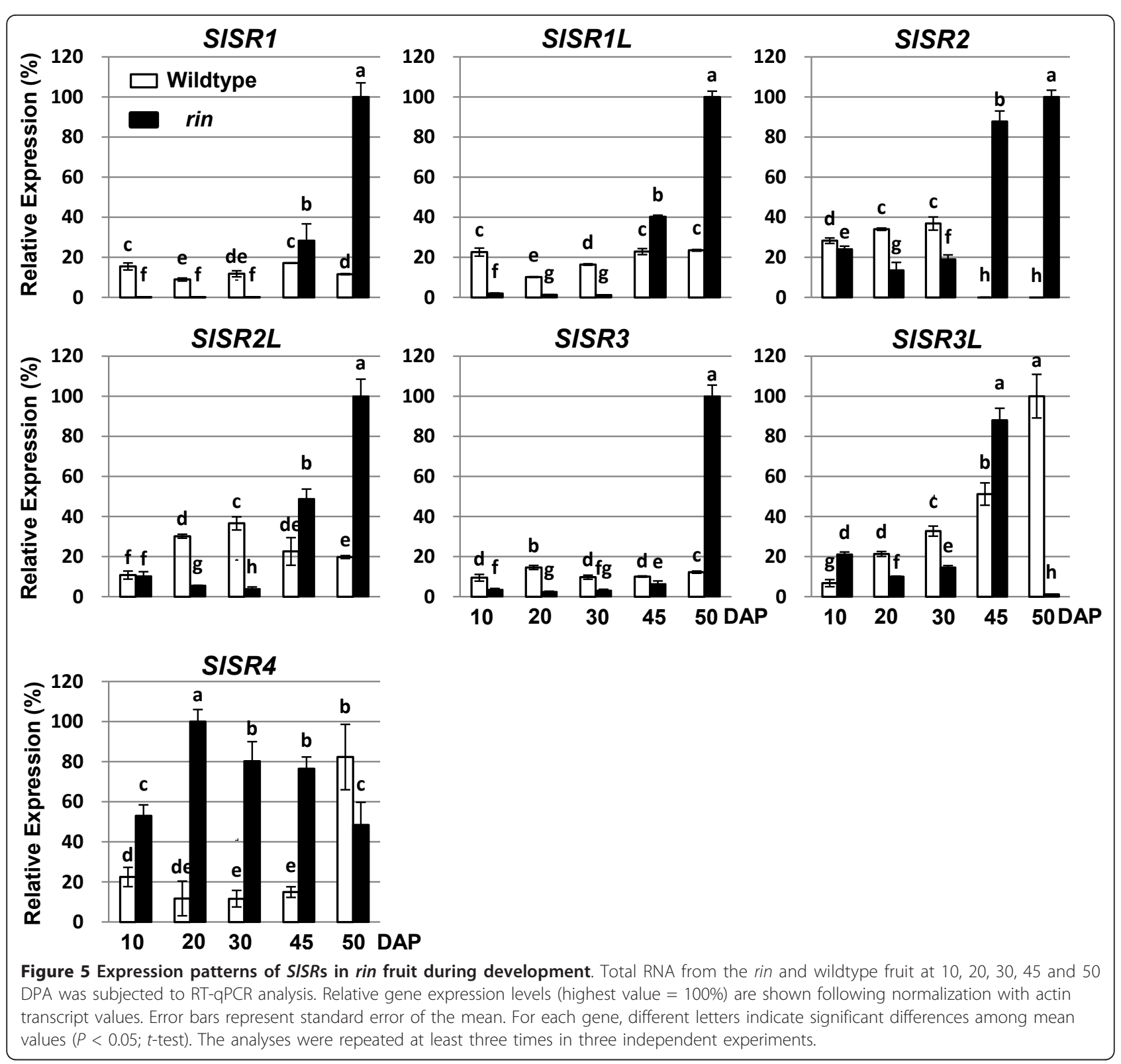

\section{Discussion}

In this study we isolated seven $S R / C A M T A$ orthologs from tomato, designated as SlSRs, which were all shown to be expressed in fruit pericarp tissue. Analyses of SlSR expression patterns during fruit development and ripening demonstrated that the expression levels of these genes are developmentally regulated. In addition, it was shown that their expression is stimulated by ethylene. Fruit ripening is a complex developmental process, and in climacteric fruits such as tomato, ripening is controlled by a combination of developmental signals and the gaseous hormone ethylene. Characterization of tomato spontaneous ripening mutants has helped to elucidate control elements and regulatory pathways involved in climacteric ripening in this useful model fruit. $N r$ was the first cloned ripening gene and was shown to encode an ethylene receptor [38]. The mutation of $\mathrm{Nr}$ affects the ethylene perception and hence blocks fruit ripening. $\mathrm{Gr}$ is another gene associated with ethylene signalling [41]. On other hand, Rin, Nor and $\mathrm{Cnr}$ were were all found to encode transcription factors or a protein regulating transcription $[39,40]$. The rin mutation is especially interesting since it has been used to breed many commercial tomato hybrids which exhibit slow fruit ripening and long shelf life. RIN codes for a MADS-box protein of the SEPALLATA clade [39]. Homologues of RIN have been found to be expressed in grape [44] and strawberry [45] and others, thus 


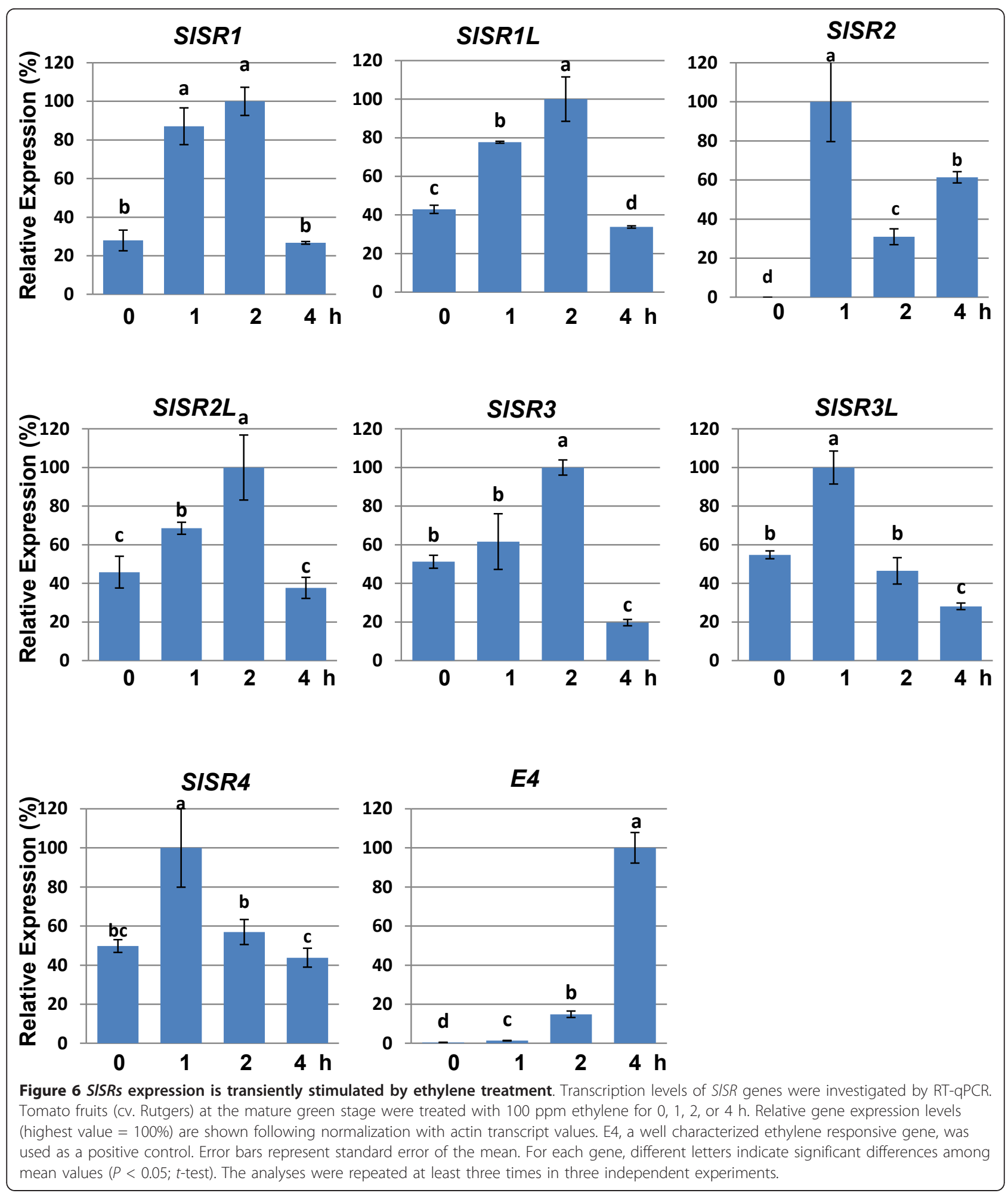

suggesting a possible involvement in fruit development and ripening in both climacteric and non-climacteric fruits. In tomato, both rin and Nor fruits are responsive to ethylene, as shown by monitoring expression of ethylene-inducible genes. However, neither mutant ripens after ethylene treatment, suggesting that both genes are located upstream of crucial ripening activities (including ethylene production) and function in an 
ethylene-independent manner [3,39]. It is still unclear what the hierarchy is between these transcription networks and how the ethylene-dependent and ethyleneindependent pathways coordinate to control the ripening process. Our results show that the expression patterns of SlSRs are altered in rin, Nor and $\mathrm{Nr}$ mutants compared with wildtype fruit, and the most significant changes occur in rin fruit. This raises the possibility that SlSRs are downstream targets of RIN. However, since ethylene treatment can stimulate SISR expression, SISRs could be regulated by both the ethylene-independent developmental network and ethylene-mediated signaling.

Calcium is recognized as a universal second messenger, which mediates responses to external stimuli and hormonal changes in plants via calcium sensors such as calmodulin [14,16,18-23]. Calcium treatment has been used to delay fruit ripening and senescence, and to maintain fruit quality $[5-7,9,46]$. Like all other $S R / C A M$ $T A$ s that have been characterized, the seven SlSRs encode a type of calcium/calmodulin-binding transcription factor. It has been reported that calcium/calmodulin-binding to SR/CAMTAs in Arabidopsis and Drosophila is crucial for their in vivo functions $[29,47,48]$. Therefore, it can reasonably be speculated that calcium regulates fruit development and ripening by forming a calcium/calmodulin complex to activate SISRs, which then modulate the expression of downstream genes. Possibly, SISRs serve as a coordinator for multiple signaling pathways involved in the regulation of fruit development and ripening. Similar to RIN, SR/ $C A M T A$ is present in both climacteric and non-climacteric fruits, and therefore could function during non-climacteric fruit development and ripening as well.

Based on their expression patterns, SlSRs share some similarities yet exhibit substantial differences as well. Similarities include their positive responsiveness to ethylene treatment and their dramatically altered expression levels in rin mutant compared with wildtype fruit. However, the seven SlSRs exhibit different expression patterns during fruit development and ripening, suggesting that while they have some redundancy, individual genes may have specific functions. Interestingly, expression of SISR3L, a fruit specific gene, is suppressed at the breaker equivalent stage (50 DPA) in rin mutant fruit. Conversely, other SlSRs, except SlSR4, show significantly increased expression at the breaker stage in rin compared with wildtype fruit, suggesting that SISR expression is regulated positively and negatively by the RINmediated fruit ripening network. It is noteworthy that SISR2 exhibited a uniquely different expression pattern in developing fruit when compared with the other six SISRs, i.e. high expression during fruit enlargement, complete suppression during the mature green and breaker stages, and rapid induction with ripening after breaker. A similar expression pattern was reported for the tomato $\beta$-galactosidase gene TBG5 [49]. $\beta$-Galactosidase is important for hemicellulosic modifications that occur during cell division, cell growth and fruit ripening. It will be interesting to investigate whether there is any relationship between SISR2 and cell wall modification.

A prior study showed that SR/CAMTAs specifically recognize the CGCG-box cis-element [26]. For example, AtSR1, an Arabidopsis SR/CAMTA ortholog, targets the CGCG-box in the promoter of EDS1, a key gene for the biosynthesis of salicylic acid [29]. Salicylic acid has been shown to down-regulate the expression of ACC synthase and thereby reduce ethylene biosynthesis [30,31], and to also delay fruit ripening [50,51]. Based on microarray assays, other possible targets identified for AtSR1 include genes encoding expansin, $\beta$-1,3-glucanase, phospholipase A2, accelerated cell death protein 6 , and senescence associated protein 21 [33]. Assuming that SISRs similarly regulate gene expression via binding to CGCG-box cis-elements, it is possible to identify SISRs targets by searching those tomato genes carrying CGCG-box(s) in their promoter regions.

\section{Conclusions}

We have isolated seven SlSR genes, the tomato SR/ CAMTA orthologs. The expression levels of SlSRs are differentially regulated mainly by development signals, as well as by ethylene. Importantly, SlSR expression patterns during fruit development are altered in rin, a tomato ripening mutant, suggesting that SlSRs are located downstream of the Rin-regulated network. SlSRs encode calcium/camodulin-regulated transcription factors. Our data suggest that SlSRs act as a signal node candidate connecting developmental, ethylene-mediated and calcium-mediated signals, thereby regulating fruit development and ripening. Further functional studies of SISRs will help to elucidate the signaling networks involved in these processes.

\section{Methods}

\section{Plant materials and treatments}

Tomato plants (Solanum lycopersicum cv Rutgers) were grown in a greenhouse at $28^{\circ} \mathrm{C}$ with a $16 \mathrm{~h} / 8 \mathrm{~h}$ (light/ dark) photoperiod. The ripening mutants rin, Nor and $\mathrm{Nr}$ were all in the cv Rutgers background. Flowers were tagged at anthesis and manually pollinated, and fruit were harvested according to the number of DPA (days post anthesis) or based on their surface color using ripening stages set by USDA. The equivalent stages to mature green and breaker stages for three ripening mutants are 45 and 50 DPA, respectively [49].

Fruit used in ethylene treatment experiments were harvested at the mature green stage and incubated under ambient conditions overnight to reduce harvest 
shock. Thereafter the fruit were sealed in a jar with 100 ppm ethylene for different time periods. Pericarp tissue excised from the fruit was frozen in liquid nitrogen and stored in $-80^{\circ} \mathrm{C}$.

\section{Cloning of tomato SR/CAMTA genes}

Six Arabidopsis SR/CAMTA orthologs were used to search tomato EST clone collections and Unigene database http://solgenomics.net/tools/blast/index.pl. The primers used for cloning SlSRs were designed from the 5' ends and 3' ends of putative coding regions according to the linear alignment to Arabidopsis genes. For those cDNA fragments lacking 5' and/or 3' ends of the coding regions, 5'-RACE and/or 3'-RACE were performed using the 5' and/or 3' RACE kit (Invitrogen, USA) to obtain the missing regions following the manufacturer's instruction. SlSRs were amplified by PCR using $P f x$ DNA polymerase (Invitrogen, USA) from the mix of cDNAs from different tomato tissues and fruit at different stages described below using gene specific primers (Table 1). The PCR fragments were cloned in a TA cloning kit (Invitrogen, USA) after adding adenine and sequenced from both ends.

\section{RNA extraction and RT-qPCR}

Total RNA was isolated from frozen tissue using the RNeasy Plant Mini Kit following the manufacturer's instructions (Qiagen, USA). After DNase digestion, the absorbance at $260 \mathrm{~nm}$ was measured using a nanodrop spectrophotometer to ensure equal amount of RNA used in the cDNA synthesis reactions among samples. One $\mu \mathrm{g}$ of total RNA was used to synthesize cDNA with the oligo- $(\mathrm{dT})_{18}$ primer following the instructions of the Superscript III kit (Invitrogen, USA). Quantitative RealTime PCR (RT-qPCR) analysis of cDNA was performed on a CFX96 Real-Time System (Bio-RAD, USA) using SYBR Green detection chemistry. Gene specific primers were designed with Primer3 software http://frodo.wi.mit. edu/primer3/. These primers are listed in Table 1 . The efficiency coefficient $\mathrm{E}$ was calculated for all primer pairs individually by plotting the relationship between $\mathrm{Ct}$ value (threshold cycle) and $\log [\mathrm{cDNA}]$. The following thermal cycle conditions were used: $95^{\circ} \mathrm{C}$ for $2 \mathrm{~min}$, followed by 45 cycles of $95^{\circ} \mathrm{C}$ for $5 \mathrm{~s}$ and $60^{\circ} \mathrm{C}$ for $20 \mathrm{~s}$. All reactions were performed in triplicate from three independent samples. Following PCR, a melting curve analysis was performed. Relative quantification of specific mRNA levels were analyzed using the cycle threshold (Ct) $2^{\Delta \Delta C t}$ method. Relative expression levels are normalized using the housekeeping gene actin (accession number: X55749) and shown in percentage (highest value $=100 \%)$. Student's $t$ test $\left(\mathrm{P}_{0.05}\right)$ was used to determine the significant difference of relative expression of individual genes among different tissues and fruit developmental stages, or wildtype and mutant fruit or ethylene treatments (Microsoft Excel 2007).

\section{Phylogenetic analysis}

Multiple sequence alignments of the encoded full-length SR/CAMTA proteins were assembled with the Geneious software package http://www.geneious.com/. Phylogenetic reconstruction was obtained by the neighboringjoining method. Genetic distances were calculated as substitutions per site using the Jukes-Cantor model.

\section{Calmodulin overlay assay}

The template coding for SISR3 were produced by PCR amplification from the cDNA with SISR3-specific oligonucleotides containing appropriate restriction sites (NdeI at the 5'-end and BamHI at the 3'-end) for cloning into the downstream of the $\mathrm{His}_{6}$ tag in a pET-30b expression vector (Novagen, USA). The nucleotide sequence of the cloned fragment produced by PCR amplification was determined after cloning into the pET-30b vector, sequencing from both sides of the pET30b cloning sites. The recombinant SISR3 was expressed in Escherichia coli strain BL21(DE3) pLysS. Proteins were separated by SDS-polyacrylamide gel electrophoresis (SDS-PAGE), electrotransferred onto a polyvinylidene difluoride membrane (Millipore), and treated with a biotinylated calmodulin with $1 \mathrm{mM} \mathrm{CaCl}_{2}$ or $2 \mathrm{mM}$ EGTA as previously described [24].

\section{Peptide binding to calmodulin}

The synthetic peptides were prepared in Genemed Synthesis Inc. Samples containing $240 \mathrm{pmol}(4 \mu \mathrm{g})$ of bovine calmodulin (Sigma) and different amounts of purified synthetic peptides in $100 \mathrm{~mm}$ Tris- $\mathrm{HCl}(\mathrm{pH}$ 7.2) and either $1 \mathrm{mM} \mathrm{CaCl}$ or $2 \mathrm{mM} \mathrm{EGTA}$ in a total volume of $30 \mu \mathrm{l}$ were incubated for $1 \mathrm{~h}$ at room temperature. The samples were analyzed by nondenaturing PAGE as described [24].

\section{SISRs accession numbers}

Accession numbers of tomato SISR genes deposited in GenBank: SlSR1, GU170838; SlSR1L, JN558810; SlSR2, JN566047; SlSR2L, JN566048; SlSR3, JN566049; SlSR3L, JN566051; SlSR4, JN566050.

\section{Additional material}

\footnotetext{
Additional file 1: Amino acid sequence alignment of SISRs with Arabidopsis orthologs. The conserved DNA-binding region near the Nterminus, ankyrin repeats in the middle and calcium/calmodulin-binding site near the C-terminus are underlined. The corresponding GenBank CDNA accession numbers or gene identification numbers are as follows: SISR1, GU170838; SISR1L, JN558810; SISR2, JN566047; SISR2L, JN566048; SISR3, JN566049; SISR3L, JN566051; SISR4, JN566050; AtSR1, AT2G22300;
} 
AtSR2, At5G09410; AtSR3, At3G16940; AtSR4, At5G64220; AtSR5, At1G67310; AtSR6, At4G16150.

Additional file 2: Calcium/calmodulin binding to peptides from SISR1, SISR2/SISR2L, and SISR4. Three peptides corresponding to the putative calmodulin-binding domains of SISR1, SISR2/SISR2L, and SISR4 were synthesized. Gel mobility shift assay showing that calcium-loaded calmodulin binds to all three peptides in the presence of calcium. Arrows indicate the positions of free calmodulin (CaM) and the peptidecalmodulin complexes.

\section{Abbreviations}

CaM: calmodulin; DPA: days post anthesis; EGTA: ethylene glycol-bis(2aminoethylether)- $N, N, N^{\prime}, N^{\prime}$-tetraacetic acid; IPTG: isopropyl $\beta-D-1-$ thiogalactopyranoside; Nor, tomato non-ripening mutant; $\mathrm{Nr}$, tomato neverripe mutant; RACE, rapid amplification of $C D N A$ ends; rin, tomato ripening inhibitor mutant; SDS-PAGE: sodium dodecyl sulfate polyacrylamide gel electrophoresis.

\section{Acknowledgements}

We would like to thank UC Davis Tomato Genetic Resources Center for providing the seeds of the rin, Nor and $\mathrm{Nr}$ mutants and cv Alisa Craig. We also thank Norman Livsey for excellent technical assistance.

\section{Authors' contributions}

TY conceived the study, designed all the experiments and performed in silicon and biochemical analyses. HP carried out all the gene expression studies and statistical analysis. TY, BDW and WSC interpreted the experimental dada and participated in writing the manuscript. All the authors read and approved the final manuscript.

Received: 22 September 2011 Accepted: 13 February 2012 Published: 13 February 2012

\section{References}

1. Matas AJ, Gapper NE, Chung MY, Giovannoni JJ, Rose JKC: Biology and genetic engineering of fruit maturation for enhanced quality and shelflife. Curr Opin Biotech 2009, 20(2):197-203.

2. Gillaspy $G$, Bendavid H, Gruissem W: Fruits - a developmental perspective. Plant Cell 1993, 5(10):1439-1451.

3. Giovannoni JJ: Genetic regulation of fruit development and ripening. Plant Cell 2004, 16:S170-S180.

4. Abbott JA, Conway WS, Sams CE: Postharvest calcium-chloride infiltration affects textural attributes of apples. J Am Soc Hortic Sci 1989, 114(6):932-936.

5. Conway WS, Sams CE: The effects of postharvest infiltration of calcium, magnesium, or strontium on decay, firmness, respiration, and ethylene production in apples. J Am Soc Hortic Sci 1987, 112(2):300-303.

6. Martin-Diana AB, Rico D, Frias JM, Barat JM, Henehan GTM, Barry-Ryan C Calcium for extending the shelf life of fresh whole and minimally processed fruits and vegetables: a review. Trends Food Sci Tech 2007, 18(4):210-218

7. Park S, Cheng NH, Pittman JK, Yoo KS, Park J, Smith RH, Hirschi KD: Increased calcium levels and prolonged shelf life in tomatoes expressing Arabidopsis H+/Ca2+ transporters. Plant Physiol 2005, 139(3):1194-1206.

8. Poovaiah BW: Role of calcium in prolonging storage life of fruits and vegetables. Food Technol-Chicago 1986, 40(5):86-89.

9. Raese JT, Drake SR: Effects of preharvest calcium sprays on apple and pear quality. J Plant Nutr 1993, 16(9):1807-1819.

10. Saftner RA, Bai JH, Abbott JA, Lee YS: Sanitary dips with calcium propionate, calcium chloride, or a calcium amino acid chelate maintain quality and shelf stability of fresh-cut honeydew chunks. Postharvest Biol Tec 2003, 29(3):257-269.

11. Demarty M, Morvan C, Thellier M: Calcium and the cell-wall. Plant Cell Environ 1984, 7(6):441-448.

12. Liu $H$, Chen FS, Yang $H S$, Yao $Y Z$, Gong $X Z$, Xin $Y$, Ding $C H$ : Effect of calcium treatment on nanostructure of chelate-soluble pectin and physicochemical and textural properties of apricot fruits. Food Res Int 2009, 42(8):1131-1140.
13. Poovaiah BW, Reddy ASN: Calcium messenger system in plants. Crit Rev Plant Sci 1987, 6(1):47-103.

14. Reddy AS: Calcium: silver bullet in signaling. Plant Sci 2001, 160(3):381-404.

15. Sanders D, Pelloux J, Brownlee C, Harper JF: Calcium at the crossroads of signaling. Plant Cell 2002, 14(Suppl):S401-S417.

16. Trewavas AJ, Malho R: Ca2+ signalling in plant cells: the big network! Cur Opin Plant Biol 1998, 1(5):428-433.

17. Xiong L, Schumaker KS, Zhu JK: Cell signaling during cold, drought, and salt stress. Plant Cell 2002, 14(Suppl):S165-S183.

18. Snedden W, Fromm H: Calmodulin as a versatile calcium signal tranducer in plants. New Physilogist 2001, 151:35-66.

19. Reddy ASN, Ali GS, Celesnik H, Day IS: Coping with stresses: roles of calcium- and calcium/calmodulin-regulated gene expression. Plant Cell 2011, 23(6):2010-2032.

20. Luan S, Kudla J, Rodriguez-Concepcion M, Yalovsky S, Gruissem W: Calmodulins and calcineurin B-like proteins: Calcium sensors for specific signal response coupling in plants. Plant Cell 2002, 14:S389-S400.

21. Bouche N, Yellin A, Snedden WA, Fromm H: Plant-specific calmodulinbinding proteins. Annu Rev Plant Biol 2005, 56:435-466.

22. Yang T, Poovaiah BW: Calcium/calmodulin-mediated signal network in plants. Trends Plant Sci 2003, 8(10):505-512.

23. Zielinski RE: Calmodulin and calmodulin-binding proteins in plants. Annu Rev Plant Physiol Plant Mol Biol 1998, 49:697-725

24. Yang TB, Poovaiah BW: An early ethylene up-regulated gene encoding a calmodulin-binding protein involved in plant senescence and death. $J$ Biol Chem 2000, 275(49):38467-38473.

25. Zegzouti H, Jones B, Frasse P, Marty C, Maitre B, Latche A, Pech JC, Bouzayen M: Ethylene-regulated gene expression in tomato fruit: characterization of novel ethylene-responsive and ripening-related genes isolated by differential display. Plant J 1999, 18(6):589-600.

26. Yang T, Poovaiah BW: A calmodulin-binding/CGCG box DNA-binding protein family involved in multiple signaling pathways in plants. $J$ Biol Chem 2002, 277(47):45049-45058

27. Bouche N, Scharlat A, Snedden W, Bouchez D, Fromm H: A novel family of calmodulin-binding transcription activators in multicellular organisms. $J$ Biol Chem 2002, 277(24):21851-21861.

28. Reddy ASN, Reddy VS, Golovkin M: A calmodulin binding protein from Arabidopsis is induced by ethylene and contains a DNA-binding motif. Biochem Bioph Res Co 2000, 279(3):762-769.

29. Du L, Ali GS, Simons KA, Hou J, Yang T, Reddy AS, Poovaiah BW: Ca(2 + )/calmodulin regulates salicylic-acid-mediated plant immunity. Nature 2009, 457(7233):1154-1158.

30. Lee S, Hong JC, Jeon WB, Chung YS, Sung S, Choi D, Joung YH, Oh BJ: The salicylic acid-induced protection of non-climacteric unripe pepper fruit against Colletotrichum gloeosporioides is similar to the resistance of ripe fruit. Plant Cell Rep 2009, 28(10):1573-1580.

31. Wang LJ, Chen SJ, Kong WF, Li SH, Archbold DD: Salicylic acid pretreatment alleviates chilling injury and affects the antioxidant system and heat shock proteins of peaches during cold storage. Postharvest Biol Tec 2006, 41(3):244-251.

32. Doherty CJ, Van Buskirk HA, Myers SJ, Thomashow MF: Roles for Arabidopsis CAMTA transcription factors in cold-regulated gene expression and freezing tolerance. Plant Cell 2009, 21(3):972-984.

33. Galon Y, Nave R, Boyce JM, Nachmias D, Knight MR, Fromm H: Calmodulinbinding transcription activator (CAMTA) 3 mediates biotic defense responses in Arabidopsis. FEBS Lett 2008, 582(6):943-948

34. Kaplan B, Davydov O, Knight H, Galon Y, Knight MR, Fluhr R, Fromm H: Rapid transcriptome changes induced by cytosolic Ca2+ transients reveal ABRE-related sequences as $\mathrm{Ca} 2+$-responsive cis elements in Arabidopsis. Plant Cell 2006, 18(10):2733-2748.

35. Walley JW, Coughlan S, Hudson ME, Covington MF, Kaspi R, Banu G, Harmer SL, Dehesh K: Mechanical stress induces biotic and abiotic stress responses via a novel cis-element. Plos Genet 2007, 3(10):1800-1812.

36. Fernandez Al, Viron $N$, Alhagdow $M$, Karimi $M$, Jones $M$, Amsellem $Z$, Sicard A, Czerednik A, Angenent G, Grierson D, et al: Flexible tools for gene expression and silencing in tomato. Plant Physiol 2009, 151(4):1729-1740

37. Giovannoni JJ: Fruit ripening mutants yield insights into ripening control. Curr Opin Plant Biol 2007, 10(3):283-289. 
38. Wilkinson JQ, Lanahan MB, Yen HC, Giovannoni JJ, Klee HJ: An ethyleneInducible component of signal-transduction encoded by Never-ripe. Science 1995, 270(5243):1807-1809.

39. Vrebalov J, Ruezinsky D, Padmanabhan V, White R, Medrano D, Drake R, Schuch W, Giovannoni J: A MADS-box gene necessary for fruit ripening at the tomato ripening-inhibitor (Rin) locus. Science 2002, 296(5566):343-346.

40. Seymour GB, Manning K, Tor M, Poole M, Hong Y, Thompson AJ, King GJ, Giovannoni JJ: A naturally occurring epigenetic mutation in a gene encoding an SBP-box transcription factor inhibits tomato fruit ripening. Nat Genet 2006, 38(8):948-952.

41. Barry CS, Giovannoni JJ: Ripening in the tomato Green-ripe mutant is inhibited by ectopic expression of a protein that disrupts ethylene signaling. P Natl Acad Sci USA 2006, 103(20):7923-7928.

42. Arazi T, Baum G, Snedden WA, Shelp BJ, Fromm H: Molecular and biochemical-analysis of calmodulin interactions with the calmodulinbinding domain of plant glutamate-decarboxylase. Plant Physiol 1995, 108(2):551-561.

43. Montgomery J, Goldman S, Deikman J, Margossian L, Fischer RL: Identification of an ethylene-responsive region in the promoter of a fruit ripening gene. P Natl Acad Sci USA 1993, 90(13):5939-5943.

44. Boss PK, Vivier M, Matsumoto S, Dry IB, Dry MR: A cDNA from grapevine (Vitis vinifera L.), which shows homology to AGAMOUS and SHATTERPROOF, is not only expressed in flowers but also throughout berry development. Plant Mol Biol 2001, 45(5):541-553.

45. Seymour GB, Ryder CD, Cevik V, Hammond JP, Popovich A, King GJ, Vrebalov J, Giovannoni JJ, Manning K: A SEPALLATA gene is involved in the development and ripening of strawberry (FragariaXananassa Duch.) fruit, a non-climacteric tissue. J Exp Bot 2011, 62(3):1179-1188.

46. Poovaiah BW: Role of calcium in ripening and senescence. Commun Soil Sci Plan 1979, 10(1-2):83-88.

47. Gong P, Han J, Reddig K, Li HS: A potential dimerization region of dCAMTA is critical for termination of fly visual response. J Biol Chem 2007, 282(29):21253-21258

48. Han JH, Gong P, Reddig K, Mitra MY, Guo P, Li HS: The fly CAMTA transcription factor potentiates deactivation of rhodopsin, a $\mathrm{G}$ proteincoupled light receptor. Cell 2006, 127(4):847-858.

49. Smith DL, Gross KC: A family of at least seven beta-galactosidase genes is expressed during tomato fruit development. Plant Physiol 2000, 123(3):1173-1183.

50. Valero D, Diaz-Mula HM, Zapata PJ, Castillo S, Guillen F, Martinez-Romero D, Serrano M: Postharvest treatments with salicylic acid, acetylsalicylic acid or oxalic acid delayed ripening and enhanced bioactive compounds and antioxidant capacity in sweet cherry. J Agr Food Chem 2011, 59(10):5483-5489.

51. Asghari M, Aghdam MS: Impact of salicylic acid on post-harvest physiology of horticultural crops. Trends Food Sci Tech 2010, 21(10):502-509.

doi:10.1186/1471-2229-12-19

Cite this article as: Yang et al:: Characterization of a calcium/ calmodulin-regulated SR/CAMTA gene family during tomato fruit development and ripening. BMC Plant Biology 2012 12:19.

\section{Submit your next manuscript to BioMed Central and take full advantage of:}

- Convenient online submission

- Thorough peer review

- No space constraints or color figure charges

- Immediate publication on acceptance

- Inclusion in PubMed, CAS, Scopus and Google Scholar

- Research which is freely available for redistribution

Submit your manuscript at www.biomedcentral.com/submit
Biomed Central 